Urologe 2009 · 48:349

DOI 10.1007/s00120-009-1942-3

Online publiziert: 21. Februar 2009

(c) Springer Medizin Verlag 2009

S. Krege ${ }^{1} \cdot$ P. Albers ${ }^{2}$

${ }^{1}$ Klinik für Urologie und Kinderurologie, Krankenhaus Maria-Hilf GmbH, Krefeld

${ }^{2}$ Klinik für Urologie, Universität Düsseldorf

\title{
Hodentumor: Standards einhalten!
}

nimmt Prof. Dr. Beyer (Leiter der Hämatoonkologie, Urban-Krankenhaus, Berlin) Stellung.

Die Therapie beim Hodentumor ist gerade in den fortgeschritteneren Stadien für den Organismus sehr belastend. Zahlreiche Akuttoxizitäten können auftreten. Da die Patienten jung sind, leiden sie aber evtl. auch unter Spättoxizitäten. Auf diesem Gebiet hat die Arbeitsgruppe um Fr. Prof. Fossa und Prof. Oldenburg (Norwegen) umfangreiches Datenmaterial ermittelt, welches von Prof. Oldenburg dargestellt und erläutert wird.

Hodentumoren haben auch im Kindesalter einen Häufigkeitsgipfel. Kindliche Hodentumoren unterscheiden sich aber in wesentlichen Punkten von denen im Erwachsenenalter. Hierzu geben Prof. Steffens (Chefarzt, Urologische Klinik, St. Antonius-Hospital, Düren) und Fr. PD Dr. Calaminus (Oberärztin, Kinderklinik, Universitätsklinikum Münster) mit Schwerpunkt pädiatrische Onkologie eine Übersicht.

Da die Realität leider immer wieder zeigt, dass die Therapieempfehlungen für den Hodentumor nicht eingehalten werden, wurde von der interdisziplinären Hodentumorgruppe Deutschlands ein Projekt zu Zweitmeinungszentren etabliert. PD Dr. Schrader (Oberarzt, Urologische Klinik, Charité Mitte, Berlin) und Fr. Szostak (Dokumentarin des Projekts) erläutern dieses und stellen eine erste Zwischenanalyse vor. Hodentumorzentren sind in anderen Ländern schon länger etabliert. Prof. Clark (Urologische Klinik, Christie Hospital, Manchester) beschreibt das englische Model.

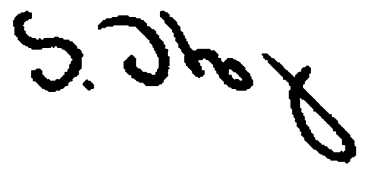

Susanne Krege

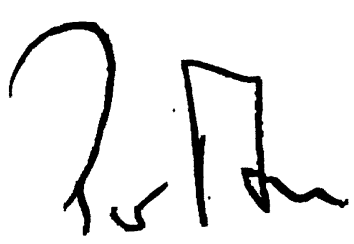

Peter Albers

\section{Korrespondenzadresse \\ PD Dr. S. Krege}

Klinik für Urologie und Kinderurologie, Krankenhaus Maria-Hilf GmbH.

Oberdießemer Straße 136, 47805 Krefeld susanne.krege@maria-hilf.de 